\title{
Generalized Fokker-Planck equation, Brownian motion, and ergodicity
}

\author{
A.V. Plyukhin \\ Department of Physics and Engineering Physics, \\ University of Saskatchewan, Saskatoon, SK S7N 5E2, Canada
}

(Dated: October 24, 2018)

\begin{abstract}
Microscopic theory of Brownian motion of a particle of mass $M$ in a bath of molecules of mass $m \ll$ $M$ is considered beyond lowest order in the mass ratio $m / M$. The corresponding Langevin equation contains nonlinear corrections to the dissipative force, and the generalized Fokker-Planck equation involves derivatives of order higher than two. These equations are derived from first principles with coefficients expressed in terms of correlation functions of microscopic force on the particle. The coefficients are evaluated explicitly for a generalized Rayleigh model with a finite time of moleculeparticle collisions. In the limit of a low-density bath, we recover the results obtained previously for a model with instantaneous binary collisions. In general case, the equations contain additional corrections, quadratic in bath density, originating from a finite collision time. These corrections survive to order $(m / M)^{2}$ and are found to make the stationary distribution non-Maxwellian. Some relevant numerical simulations are also presented.
\end{abstract}

PACS numbers: 05.40.-a, 05.20.-y

\section{INTRODUCTION}

This paper is concerned with the derivation and some properties of the generalized Fokker-Planck equation (FPE) for the distribution function $f(p, t)$ of a single stochastic variable $p$, which differs from the conventional FPE by involving $p$-derivatives of order higher than two,

$$
\frac{\partial f(p, t)}{\partial t}=\sum_{n=1}^{k>2} \frac{\partial^{n}}{\partial p^{n}} c_{n}(p) f(p, t) .
$$

Such an equation may appear as a result of a high-order truncation of the Kramers-Moyal expansion of the Markovian master equation [1],

$$
\begin{aligned}
\frac{\partial f(p, t)}{\partial t} & =\int d p^{\prime}\left\{f\left(p^{\prime}, t\right) w\left(p^{\prime} \rightarrow p\right)-f(p, t) w\left(p \rightarrow p^{\prime}\right)\right\} \\
& =\sum_{n=1}^{\infty} \frac{1}{n !}\left(-\frac{\partial}{\partial p}\right)^{n}\left\{\alpha_{n}(p) f(p, t)\right\} .
\end{aligned}
$$

In general, the naive truncation of this expansion is not a legitimate procedure, but if a problem at hand involves a small parameter $\lambda$, one can approximate the expansion by finite number of terms using an appropriate perturbation technique [2]. In case of Brownian motion of a heavy particle of mass $M$ in a thermal bath of light molecules of mass $m$ and temperature $T$, the appropriate small parameter is the mass ratio $\lambda^{2}=m / M$. In this case, to order $\lambda^{2}$ one recovers for the particle's momentum $p$ the conventional second-order FPE,

$$
\frac{\partial f(p, t)}{\partial t}=\left\{a_{1} \frac{\partial}{\partial p} p+a_{2} \frac{\partial^{2}}{\partial p^{2}}\right\} f(p, t)
$$

while going beyond order $\lambda^{2}$ leads to an equation of the form (1).

In rare cases when transition rates $w$ in the master equation (2) are known explicitly, the derivation of the generalized FPE (1) is fairly straightforward 2, 3, 4, 5], otherwise it is difficult. A popular approach engages the assumption that the fluctuating force in the corresponding Langevin equation is a Gaussian process. In this case perturbation analysis is not needed since the terms with derivatives of order higher than two vanish identically and one arrives at the conventional FPE (3), which in this case is exact. However, the assumption of Gaussian random force, although might seem physically reasonable, should not be taken for granted. In particular, for a Brownian particle it is justified only to order $\lambda^{2}$, while corrections of higher orders are essentially non-Gaussian and lead to a FPE in the generalized form (1). This paper is focused on the generalized FPE for the Brownian particle's momentum $p$ to order $\lambda^{4}$ which involves $p$-derivatives up to order four.

Despite a few important contributions (see below), the theory of Brownian motion beyond the lowest approximation did not attract much attention in the past, perhaps because of the common belief that higher order corrections are 
of little importance. However, in recent years the problem has caught some new interest. Beyond lowest order in $\lambda$ the Langevin equation for a Brownian particle involves nonlinear dissipative terms, and thus corresponds to the description beyond the level of linear response theory. One might hope that the nonlinear Langevin equation and the corresponding generalized FPE would enable to capture subtle effects of the interplay of noise and nonlinearity, which are completely washed out when one uses the conventional FPE or the corresponding linear Langevin equation. This indeed has been demonstrated for a number of problems including Brownian motors [6, 7, 8, [9] and barrier crossing [10].

The main difficulty related to the generalized FPE and the corresponding nonlinear Langevin equation is that these equations usually can not be constructed on a purely phenomenological basis. There is a substantial mathematical literature on the Langevin equation with nonlinear dissipation terms. However, for processes with nonlinear dissipation thermodynamics provides no hints about the form of fluctuation-dissipation relations, and little progress can be made without such relations. Furthermore, an attempt to go beyond the comfortable but artificial assumption of a Gaussian random force leaves one, within a phenomenological framework, with no clue how to handle correlations higher than of second order.

These difficulties suggest to derive the generalized FPE from as close to first principles as possible. A successful example of such derivation is the van Kampen's method of system size expansion [2] and in particular its application for the Rayleigh model of Brownian motion [3, 4, 5]. In this model a Brownian particle of mass $M$ moves in one dimension interacting with the heat bath of ideal gas molecules of mass $m \ll M$ and temperature $T$ through instantaneous binary collisions. As was mentioned above, the relevant small parameter is the mass ratio $\lambda^{2}=m / M$. Since the momentum of the particle $P$ is on average $\lambda^{-1}$ times larger than that of a bath molecule, it is convenient to work with the scaled particles momentum $p=\lambda P$ which is of the same order as the thermal momentum of molecules of the bath $p_{T}=\sqrt{m k_{B} T}$. The Rayleigh model is truly Markovian, and the natural starting point is the master equation (2) for the distribution function $f(p, t)$, in which transition rates can be readily found explicitly under the assumption of binary particle-molecule collisions. Transforming the Kramers-Moyal expansion into the expansion in powers of $\lambda$, one derives to order $\lambda^{2}$ the conventional FPE

$$
\frac{\partial f(p, t)}{\partial t}=\lambda^{2} D_{2} f(p, t)
$$

where $D_{2}$ is a second order differential operator

$$
D_{2}=\gamma_{0}\left\{\frac{\partial}{\partial p} p+p_{T}^{2} \frac{\partial^{2}}{\partial p^{2}}\right\}, \quad \gamma_{0}=\frac{8 \nu}{\sqrt{2 \pi}} \frac{p_{T}}{m},
$$

$\nu$ is the number of bath molecules per unit length, $p_{T}=\sqrt{m / \beta}$ is the molecule's thermal momentum, and $\beta$ is the inverse temperature $\beta=1 / k_{B} T$. An extension to order $\lambda^{4}$ leads to the generalized FPE in the form

$$
\frac{\partial f(p, t)}{\partial t}=\left\{\lambda^{2} D_{2}+\lambda^{4} D_{4}\right\} f(p, t)
$$

where the operator $D_{4}$ involves derivatives up to order four,

$$
D_{4}=\gamma_{0}\left\{-\frac{\partial}{\partial p} p+\frac{1}{6 p_{T}^{2}} \frac{\partial}{\partial p} p^{3}-2 p_{T}^{2} \frac{\partial^{2}}{\partial p^{2}}+\frac{3}{2} \frac{\partial^{2}}{\partial p^{2}} p^{2}+\frac{8 p_{T}^{2}}{3} \frac{\partial^{3}}{\partial p^{3}} p+\frac{4 p_{T}^{4}}{3} \frac{\partial^{4}}{\partial p^{4}}\right\} .
$$

Note that terms of order $\lambda^{3}$ vanish due to symmetry. In what follows I will refer to Eq. (6), first obtained in [3] , as the van Kampen equation.

One can verify that the Maxwellian distribution $f_{M}(p)=C \exp \left(-\beta p^{2} / 2 m\right)$ is the stationary solution for both the standard FPE (4) and the van Kampen equation (므),

$$
D_{2} f_{M}(p)=D_{4} f_{M}(p)=0 .
$$

However, in contrast to the conventional FPE (4), the van Kampen equation (6) does not preserve the positivity of the solution and therefore can not be an exact equation for any stochastic process. Yet, as an approximation beyond lowest order in $\lambda$, the equation (6) is useful and predicts a number of qualitatively new features. For instance, while the conventional FPE (4) gives for the average momentum $\langle p(t)\rangle=\int d p f(p, t) p$ the closed equation $\langle\dot{p}\rangle=-\lambda^{2} \gamma_{0}\langle p\rangle$, the van Kampen equation (6) predicts the coupling to the third moment $\left\langle p^{3}\right\rangle$ :

$$
\frac{d}{d t}\langle p\rangle=-\lambda^{2} \gamma_{0}\left(1-\lambda^{2}\right)\langle p\rangle-\frac{1}{6} \lambda^{4} \gamma_{0} p_{T}^{-2}\left\langle p^{3}\right\rangle .
$$


For initial conditions $\langle p(0)\rangle=0$ and $\left\langle p^{3}(0)\right\rangle \neq 0$, the FPE (4) gives $\langle p(t)\rangle=0$ for any $t>0$, while the equation (9) gives nonzero average momentum for a time interval $t \sim \gamma_{0}^{-1}$. This prediction was discussed and confirmed by numerical simulation in 9]. The van Kampen equation (6) has been recently exploited in the context of rectification of thermal fluctuations [7] and to study the influence of nonlinear dissipation on the Kramers escape rate [10].

Although proved to be useful, the van Kampen equation (6) is by no means general. It is derived under assumptions similar to those for the Boltzmann equation, namely that a characteristic collision time $\tau_{c}$ is much shorter than all other relevant time scales (which implies small $\lambda$ ), and that multiple collisions are negligible (small bath's density). It is of interest to derive a generalized FPE from first principles keeping the former assumption, but relaxing the latter. Some aspects of this problem were addressed already in pioneering works on the microscopic theory of Brownian motion [11] and further developed in [12, 13, 14]. Perhaps the most elaborate work is the paper by van Kampen and Oppenheim [15]. They applied the projection operator technique directly to the Liouville equation for the total particle-bath distribution function and derived a generalized FPE of order $\lambda^{4}$. The coefficients in the equation are expressed in terms of rather complicated correlation functions, and no attempt has been made to compare the result with the van Kampen equation (6).

It was noted by Seke 16 that the projection operator method, when applied to the Liouville equation, involves some subtlety and may be inconsistent. The alternative way, which we shall follow in this paper, is to apply the projector operator technique directly to the equation of motion of the particle, to derive microscopically the Langevin equation for the particle's momentum $p$, and then to apply a standard routine [1] to construct a corresponding FPE for the distribution function $f(p, t)$.

It is generally believed that the two methods of derivation, namely "Liouville $\rightarrow$ Fokker-Planck" and "Equation of motion $\rightarrow$ Langevin $\rightarrow$ Fokker-Planck", should give the same result. To lowest order $\lambda^{2}$ it is indeed the case: both methods lead to the standard second-order FPE (3). However, we have found recently [17] that beyond the lowest order predictions of the two methods are different. Moreover, it was found that the second method (from Langevin to Fokker-Planck) leads to a generalized FPE with a rather disturbing property, namely, its stationary solution was found non-Maxwellian. The intention of this paper is to follow this line in detail to obtain a generalized FPE to order $\lambda^{4}$ in a complete form. The equation we arrive at, namely Eq.(70) in Section V, involves terms linear and quadratic in the density of bath molecules $n$. For a very diluted bath the latter can be neglected, and the equation is reduced to the van Kampen equation (6), as expected. The results related to the terms quadratic in $n$ are controversial. These terms are absent in the van Kampen equation for a binary collisions model, have the structure different than that in the van Kampen-Oppenheim approach, and make the stationary solution inconsistent with Boltzmann-Gibbs statistics. Whether these results provide a consistent proof of weak nonergodicity of Brownian motion or are merely an indication that a naive perturbation scheme does not apply beyond the Markovian approximation remains an open question. As will be discussed, a direct unambiguous verification of non-ergodic effects by numerical simulation may be a non-trivial task.

The possibility of deviations from the Maxwellian distribution has been discussed in literature for a long time. It is known that even small deviations may be important for thermally activated processes, in particular for thermonuclear reaction rates in astrophysical plasma [18]. Several mechanisms were proposed to justify such deviations, but in our opinion none of them are quite satisfactory. For instance, introducing a nonlinear dissipating term into the Langevin equation and assuming that the fluctuating force is Gaussian, one generally arrives at a second order FPE with a non-Maxwellian stationary solution [19]. However, as we already noted, nonlinear corrections to the dissipation force for a Brownian particle is of order higher than $\lambda^{2}$. In this case an accurate perturbation procedure leads to a generalized FPE of order higher than two, which is a clear indication that the assumption of Gaussian random force is not justified beyond the lowest order.

The plan of the paper is as follows. In sections II and III, the Mazur-Oppenheim version of the projection operator technique is applied to derive the nonlinear Langevin equation of order $\lambda^{4}$. In this part we mostly follow the previous paper [20] making some small yet important corrections. The corresponding generalized FPE is constructed in Section IV and analyzed in Section V. In Section VI we present the results for the generalized Rayleigh model which allows analytical evaluation of all relevant correlation functions. Also, in this section the results of numerical simulation are discussed. Summarizing remarks are collected in Section VII.

\section{NON-MARKOVIAN LANGEVIN EQUATION}

Consider a structureless Brownian particle of mass $M$ immersed in a thermal bath comprised of molecules of mass $m$. It is assumed that the mass ratio $\lambda^{2}=m / M$ is small and that the bath is initially in equilibrium at temperature $T$. The aim of this and the next sections is to derive the Langevin equation for the particle to order $\lambda^{4}$. As will be shown, such an equation involves a nonlinear correction to the damping term, which is cubic in the particle's momentum. 
The Hamiltonian of the system is

$$
\begin{aligned}
H & =\frac{P^{2}}{2 M}+H_{0} \\
H_{0} & =\sum_{i} \frac{p_{i}^{2}}{2 m}+U(x, X) .
\end{aligned}
$$

Here $x=\left\{x_{i}\right\}$ and $p_{i}$ are positions and momenta of bath molecules, $X$ and $P$ are those of the Brownian particle, $H_{0}$ is the Hamiltonian of the bath in the field of the Brownian particle fixed at $X$, and the potential $U$ describes the intermolecular interaction and the interaction between molecules and the particle. To simplify notations we shall consider a one-dimensional problem. The coupling of the particle with hydrodynamic modes of the bath will be neglected, in which case the extension to higher dimensions is simple.

As was already noted, it is convenient to work with the scaled momentum of the particle $p=\lambda P$, since this quantity on average is expected to be of the same order as the typical momentum of a bath molecule $p_{T}=\sqrt{m k_{B} T}=\sqrt{m / \beta}$. Writing the Liouville operator $\mathcal{L}$ in terms of $p$ has the advantage of extracting the small parameter $\lambda$ explicitly,

$$
\begin{aligned}
\mathcal{L} & =\mathcal{L}_{0}+\lambda \mathcal{L}_{1}, \\
\mathcal{L}_{0} & =\sum_{i}\left\{\frac{p_{i}}{m} \frac{\partial}{\partial x_{i}}+F_{i} \frac{\partial}{\partial p_{i}}\right\}, \\
\mathcal{L}_{1} & =\frac{p}{m} \frac{\partial}{\partial X}+F \frac{\partial}{\partial p} .
\end{aligned}
$$

Here $F_{i}=-\partial U / \partial x_{i}$ and $F=-\partial U / \partial X$ are the forces on the $i$-th bath molecule and on the Brownian particle, respectively.

The operator $\mathcal{L}_{0}$ corresponds to the Hamiltonian $H_{0}$ and governs dynamics of the bath in the field of the Brownian particle which is fixed at $X$. Since $M \gg m$ one might expect that the force $F(t)=e^{\mathcal{L} t} F(0)$ exerted by the bath on the particle is close to the force on a fixed particle (pressure),

$$
F(t) \approx F_{0}(t) \equiv e^{\mathcal{L}_{0} t} F(0)
$$

This intuition is implemented in the Mazur-Oppenheim approach 21] using the projection operator $\mathcal{P}$ which averages a dynamical variable $A$ over the canonical distribution $\rho=Z^{-1} \exp \left(-\beta H_{0}\right)$, for bath variables at $t=0$,

$$
\mathcal{P} A=\langle A\rangle \equiv \int \rho A \prod_{i} d x_{i} d p_{i}
$$

The idea is to decompose the total force $F(t)$ on the particle into a zero centered fluctuating ("random") and a regular ("dissipative") parts. Using the operator identity

$$
e^{(A+B) t}=e^{\mathcal{A} t}+\int_{0}^{t} d \tau e^{\mathcal{A}(t-\tau)} \mathcal{B} e^{(\mathcal{A}+B) \tau},
$$

with $\mathcal{A}=\mathcal{L}$ and $\mathcal{B}=-\mathcal{P} \mathcal{L}$, one may decompose the force $F(t)=e^{\mathcal{L} t} F(0)$ as follows

$$
F(t)=F^{\dagger}(t)+\int_{0}^{t} d \tau e^{\mathcal{L}(t-\tau)} \mathcal{P} \mathcal{L} F^{\dagger}(\tau)
$$

where $F^{\dagger}(t)=e^{\mathcal{Q L} t} F$ and $\mathcal{Q}=1-\mathcal{P}$. The factor $\mathcal{P} \mathcal{L} F^{\dagger}(\tau)$ in the integral in Eq. (18) can be simplified taking into account the orthogonality of $\mathcal{P}$ and $\mathcal{L}_{0}\left(\mathcal{P} \mathcal{L}_{0}=0\right)$, and the equality

$$
\left\langle\frac{\partial}{\partial X} F^{\dagger}(t)\right\rangle=-\beta\left\langle F(0) F^{\dagger}(t)\right\rangle
$$

which can be verified by integration by parts. As a result, one can write the equation of motion for the scaled momentum $\dot{p}(t)=\lambda F(t)$ in the form [21]

$$
\frac{d p(t)}{d t}=\lambda^{2} \int_{0}^{t} d \tau e^{\mathcal{L}(t-\tau)}\left(\frac{\partial}{\partial p}-\frac{\beta}{m} p\right)\left\langle F(0) F^{\dagger}(\tau)\right\rangle+\lambda F^{\dagger}(t),
$$


where $F^{\dagger}(t)$ is a fluctuating force obeying $\left\langle F^{\dagger}(t)\right\rangle=\mathcal{P} e^{\mathcal{Q L t}} F(0)=0$.

The equation (20) is exact but hardly instructive. To get some progress one needs to expand the fluctuating force $F^{\dagger}(t)=e^{\left(\mathcal{L}_{0}+\lambda \mathcal{Q} \mathcal{L}_{1}\right) t} F$ in powers of $\lambda$,

$$
F^{\dagger}(t)=F_{0}(t)+\lambda F_{1}(t)+\lambda^{2} F_{2}(t)+O\left(\lambda^{3}\right)
$$

Using the identity (17) one obtains

$$
\begin{aligned}
& F_{0}(t)=e^{\mathcal{L}_{0} t} F(0) \\
& F_{1}(t)=\int_{0}^{t} d t_{1} e^{\mathcal{L}_{0}\left(t-t_{1}\right)} \mathcal{Q} \mathcal{L}_{1} F_{0}\left(t_{1}\right) \\
& F_{2}(t)=\int_{0}^{t} d t_{1} e^{\mathcal{L}_{0}\left(t-t_{1}\right)} \mathcal{Q} \mathcal{L}_{1} \int_{0}^{t_{1}} d t_{2} e^{\mathcal{L}_{0}\left(t_{1}-t_{2}\right)} \mathcal{Q} \mathcal{L}_{1} F_{0}\left(t_{2}\right) .
\end{aligned}
$$

In contrast to the pressure $F_{0}(t)$, the higher order corrections $F_{1}(t)$ and $F_{2}(t)$ depend on the particle's momentum $p$. Seeking an equation for $p(t)$, one needs to extract this dependence explicitly:

$$
\begin{aligned}
F_{1}(t) & =\frac{p}{m} \int_{0}^{t} d t^{\prime}\left\{G_{1}\left(t, t^{\prime}\right)-\left\langle G_{1}\left(t, t^{\prime}\right)\right\rangle\right\} \\
F_{2}(t) & =\left(\frac{p}{m}\right)^{2} \int_{0}^{t} d t^{\prime} \int_{0}^{t^{\prime}} d t^{\prime \prime}\left\{G_{2}\left(t, t^{\prime}, t^{\prime \prime}\right)-\left\langle G_{2}\left(t, t^{\prime}, t^{\prime \prime}\right)\right\rangle\right\} \\
& +\frac{1}{m} \int_{0}^{t} d t^{\prime} \int_{0}^{t^{\prime}} d t^{\prime \prime} G_{0}\left(t-t^{\prime}\right)\left\{G_{1}\left(t, t^{\prime \prime}\right)-\left\langle G_{1}\left(t, t^{\prime \prime}\right)\right\rangle\right\} .
\end{aligned}
$$

Here the functions $G_{i}$ are defined as follows

$$
\begin{aligned}
G_{0}(t) & =F_{0}(t) \\
G_{1}\left(t, t_{1}\right) & =\mathcal{S}\left(t-t_{1}\right) F_{0}\left(t_{1}\right) \\
G_{2}\left(t, t_{1}, t_{2}\right) & =\mathcal{S}\left(t-t_{1}\right) \mathcal{S}\left(t_{1}-t_{2}\right) F_{0}\left(t_{2}\right)
\end{aligned}
$$

and the operator $\mathcal{S}$ is

$$
\mathcal{S}\left(t_{i}-t_{k}\right)=e^{\mathcal{L}_{0}\left(t_{i}-t_{k}\right)} \frac{\partial}{\partial X}
$$

The functions $G_{i}$ do not depend on the particle momentum $p$ and, as we shall see, all coefficients in the $\lambda^{4}$-order generalized FPE can be expressed in terms of correlation functions of $G_{0}, G_{1}$, and $G_{2}$.

Let us now return to the exact equation of motion Eq.(20) and expand the correlation $\left\langle F(0) F^{\dagger}(t)\right\rangle$ to order $\lambda^{2}$,

$$
\left\langle F(0) F^{\dagger}(t)\right\rangle=\left\langle F(0) F_{0}(t)\right\rangle+\lambda^{2}\left\langle F(0) F_{2}(t)\right\rangle .
$$

Note that $\left\langle F(0) F_{1}(t)\right\rangle=0$ due to symmetry. Using (23), one can write the above equation in the following form

$$
\left\langle F(0) F^{\dagger}(t)\right\rangle=C_{0}(t)+\lambda^{2}\left\{\left(\frac{p}{m}\right)^{2} C_{1}(t)+\frac{1}{m} C_{2}(t)\right\},
$$

where

$$
\begin{aligned}
& C_{0}(t)=\left\langle\left\langle G_{0}(0) G_{0}(t)\right\rangle\right\rangle, \\
& C_{1}(t)=\int_{0}^{t} d t^{\prime} \int_{0}^{t^{\prime}} d t^{\prime \prime}\left\langle\left\langle G_{0}(0) G_{2}\left(t, t^{\prime}, t^{\prime \prime}\right)\right\rangle\right\rangle, \\
& C_{2}(t)=\int_{0}^{t} d t^{\prime} \int_{0}^{t^{\prime}} d t^{\prime \prime}\left\langle\left\langle G_{0}(0) G_{0}\left(t-t^{\prime}\right) G_{1}\left(t, t^{\prime \prime}\right)\right\rangle\right\rangle .
\end{aligned}
$$

In the above equations, cumulants $\left\langle\left\langle A_{1} A_{2} \cdots A_{k}\right\rangle\right\rangle$ are defined in a usual way, i.e. as a part of the correlation $\left\langle A_{1} A_{2} \cdots A_{k}\right\rangle$ which can not be reduced to the product of correlations of lower order. We shall need correlations and 
cumulants up to order four, which are related to each other as follows:

$$
\begin{aligned}
\langle A\rangle= & \langle\langle A\rangle\rangle, \\
\left\langle A_{1} A_{2}\right\rangle= & \left\langle A_{1}\right\rangle\left\langle A_{2}\right\rangle+\left\langle\left\langle A_{1} A_{2}\right\rangle\right\rangle, \\
\left\langle A_{1} A_{2} A_{3}\right\rangle= & \left\langle A_{1}\right\rangle\left\langle A_{2}\right\rangle\left\langle A_{3}\right\rangle+\left\langle A_{1}\right\rangle\left\langle\left\langle A_{2} A_{3}\right\rangle\right\rangle+\left\langle A_{2}\right\rangle\left\langle\left\langle A_{3} A_{1}\right\rangle\right\rangle+\left\langle A_{3}\right\rangle\left\langle\left\langle A_{2} A_{1}\right\rangle\right\rangle+\left\langle\left\langle A_{1} A_{2} A_{3}\right\rangle\right\rangle, \\
\left\langle A_{1} A_{2} A_{3} A_{4}\right\rangle= & \left\langle A_{1}\right\rangle\left\langle A_{2}\right\rangle\left\langle A_{3}\right\rangle\left\langle A_{4}\right\rangle \\
& +\left\langle A_{1}\right\rangle\left\langle\left\langle A_{2} A_{3} A_{4}\right\rangle\right\rangle+\left\langle A_{2}\right\rangle\left\langle\left\langle A_{1} A_{3} A_{4}\right\rangle\right\rangle+\left\langle A_{3}\right\rangle\left\langle\left\langle A_{1} A_{2} A_{4}\right\rangle\right\rangle+\left\langle A_{4}\right\rangle\left\langle\left\langle A_{1} A_{2} A_{3}\right\rangle\right\rangle \\
& +\left\langle\left\langle A_{1} A_{2}\right\rangle\right\rangle\left\langle\left\langle A_{3} A_{4}\right\rangle\right\rangle+\left\langle\left\langle A_{1} A_{3}\right\rangle\right\rangle\left\langle\left\langle A_{2} A_{4}\right\rangle\right\rangle+\left\langle\left\langle A_{1} A_{4}\right\rangle\right\rangle\left\langle\left\langle A_{2} A_{3}\right\rangle\right\rangle+\left\langle\left\langle A_{1} A_{2} A_{3} A_{4}\right\rangle\right\rangle .
\end{aligned}
$$

The important property of cumulants of functions $G_{i}$ is that they are linear in the concentration of bath molecules $n$.

$$
\left\langle\left\langle G_{i} G_{j} \cdots G_{k}\right\rangle\right\rangle \sim n .
$$

This may be demonstrated noticing that $G_{i}$ is a linear functional of cumulants for the density of bath particles $N(z, t)=\sum_{i} \delta\left(z-z_{i}(t)\right)$, where $z_{i}$ denotes the coordinate-momentum pair $\left(x_{i}, p_{i}\right)$ of a bath particle. In turn, one can observe that cumulants $\left\langle\left\langle N\left(z_{1}, t_{1}\right) N\left(z_{2}, t_{2}\right) \ldots N\left(z_{k}, t_{k}\right)\right\rangle\right\rangle$ of any order $k$ depend linearly on the concentration of bath molecules $n$. For instance, in the expression for the product $N\left(z_{1}, t_{1}\right) N\left(z_{2}, t_{2}\right)$ one can write the double sum as $\sum_{i, j}=\sum_{i \neq j}+\sum_{i=j}$ which gives

$$
\left\langle N\left(z_{1}, t_{1}\right) N\left(z_{2}, t_{2}\right)\right\rangle=\left\langle N\left(z_{1}, t_{1}\right)\right\rangle\left\langle N\left(z_{2}, t_{2}\right)\right\rangle+\sum_{i}\left\langle\delta\left(z_{1}-z_{i}\left(t_{1}\right)\right) \delta\left(z_{2}-z_{i}\left(t_{2}\right)\right)\right\rangle .
$$

Here the second term on the right side is by definition the cumulant $\left\langle\left\langle N\left(z_{1}, t_{1}\right) N\left(z_{2}, t_{2}\right)\right\rangle\right\rangle$ and is obviously linear in $n$.

Substituting the expansion (27) into the equation of motion (20) one arrives at a nonlinear and non-Markovian Langevin equation of order $\lambda^{4}$,

$$
\frac{d p(t)}{d t}=-\lambda^{2} \int_{0}^{t} d \tau M_{1}(\tau) p(t-\tau)-\lambda^{4} \int_{0}^{t} d \tau M_{2}(\tau) p^{3}(t-\tau)+\lambda F(t) .
$$

Here the memory kernels are

$$
\begin{aligned}
& M_{1}(t)=\frac{\beta}{m} C_{0}(t)-\lambda^{2} \frac{2}{m^{2}} C_{1}(t)+\lambda^{2} \frac{\beta}{m^{2}} C_{2}(t), \\
& M_{2}(t)=\frac{\beta}{m^{3}} C_{1}(t)
\end{aligned}
$$

and the fluctuating force $\lambda F(t)$ involves three components,

$$
F(t)=F_{0}(t)+\lambda F_{1}(t)+\lambda^{2} F_{2}(t),
$$

where $F_{0}(t)$ is the fluctuating pressure, and $F_{1}(t)$ and $F_{2}(t)$ are defined by Eqs. (23). The zero-mean term $\lambda^{3} F_{3}(t)$ is discarded because to order $\lambda^{4}$ it does not contribute to correlations of the fluctuating force $\lambda F(t)$.

\section{MARKOVIAN LANGEVIN EQUATION}

The relative importance of memory effects described by the non-Markovian Langevin equation (32) depends on how fast the particle's momentum $p(t)$ evolves on the time scale $\tau_{c}$ for the decay of the memory kernels $M_{1}(t)$ and $M_{2}(t)$. In what follows we shall assume that the characteristic time $\tau_{c}$ does exist. This assumption is not satisfied, for example, for the Rubin's model (a heavy impurity embedded in the harmonic chain) where $M_{2}(t)$ is identically zero, and $M_{1}(t)$ decays with time as a power law. It is known, however, that in many cases long-tail effects are indeed negligible for sufficiently small $\lambda$, although the justification may require rather subtle argument [14, 22]

The memory kernels $M_{i}(t)$ are expressed in terms of correlation functions $C_{i}(t)$ which do not depend on $\lambda$. Then the characteristic decay time of the kernels does not depend on $\lambda$ either, $\tau_{c} \sim \lambda^{0}$. On the other hand, as follows from Eq.(32), the characteristic time for relaxation of the particle's momentum $\tau_{p}$ is of order $\lambda^{-2}$ and thus expected to be much longer than $\tau_{c}$. This suggests that the non-Markovian equation (32) can be expanded in powers of $\lambda$ about its Markovian limit.

One way to make such an expansion is to use in Eq. (32) the following substitution 21]

$$
p^{n}(t-\tau)=p^{n}(t)-\int_{t-\tau}^{t} d t^{\prime} \frac{d}{d t^{\prime}} p^{n}\left(t^{\prime}\right) .
$$


The main contributions to the integrals in Eq. the right-hand side of Eq.(35) is of order $\dot{p} \sim \lambda$. Then the nonlinear dissipative term in the Langevin equation (32) can be written in the local form

$$
-\lambda^{4} \int_{0}^{t} d \tau M_{2}(\tau) p^{3}(t-\tau)=-\lambda^{4} p^{3}(t) \int_{0}^{t} d \tau M_{2}(\tau)+O\left(\lambda^{5}\right) .
$$

To order $\lambda^{4}$ this justifies the Markovian ansatz for the nonlinear dissipative term

$$
-\lambda^{4} \int_{0}^{t} d \tau M_{2}(\tau) p^{3}(t-\tau) \rightarrow-\lambda^{4} p^{3}(t) \int_{0}^{\infty} d \tau M_{2}(\tau) .
$$

Here the upper integration limit in the right-hand side is taken to infinity since we restrict ourselves to the coarse-grain description on the time scale much longer than the characteristic time for bath fluctuations, $t \gg \tau_{c}$.

The same argument for the linear dissipative term gives

$$
-\lambda^{2} \int_{0}^{t} d \tau M_{1}(\tau) p(t-\tau)=-\lambda^{2} p(t) \int_{0}^{t} d \tau M_{0}(\tau)+O\left(\lambda^{3}\right) .
$$

In contrast to the nonlinear dissipation term, the Markovian approximation

$$
-\lambda^{2} \int_{0}^{t} d \tau M_{1}(\tau) p(t-\tau) \rightarrow-\lambda^{2} p(t) \int_{0}^{\infty} d \tau M_{0}(\tau)
$$

can be applied for the linear dissipative force only in lowest order $\lambda^{2}$, in which case one recovers the conventional linear Langevin equation

$$
\frac{d p(t)}{d t}=-\lambda^{2} \gamma_{0} p(t)+\lambda F_{0}(t)
$$

with the pressure $F_{0}(t)$ as a fluctuating force and the dissipation constant

$$
\gamma_{0}=\frac{\beta}{m} \int_{0}^{\infty} d t C_{0}(t)
$$

Let us now derive a local form for the linear dissipative term to order $\lambda^{4}$. Using again the substitution (35), one can write the linear term as a local expression plus a correction term $\Delta(t)$

$$
-\lambda^{2} \int_{0}^{t} d \tau M_{1}(\tau) p(t-\tau)=-\lambda^{2} p(t) \int_{0}^{t} d \tau M_{1}(\tau)+\Delta(t)
$$

The correction has a form

$$
\Delta(t)=\lambda^{2} \int_{0}^{t} d \tau M_{1}(\tau) \int_{t-\tau}^{t} d \tau^{\prime} \dot{p}\left(\tau^{\prime}\right)
$$

Here the essential integration range is of order $\tau_{c} \sim \lambda^{0}$, so that the correction term $\Delta \sim \lambda^{2} \dot{p} \sim \lambda^{3}$. Using the linear Langevin equation (40), one can write $\Delta$ as follows

$$
\Delta(t)=-\lambda^{4} \gamma^{\star} p(t)+\lambda^{3} F^{\star}(t)
$$

Here the first term $-\lambda^{4} \gamma^{\star} p$ with

$$
\gamma^{\star}=\left(\frac{\beta}{m}\right)^{2} \int_{0}^{\infty} d t C_{0}(t) \int_{0}^{\infty} d t C_{0}(t) t
$$

is a correction to the local linear dissipative force, while the second term

$$
F^{\star}(t)=\frac{\beta}{m} \int_{0}^{\infty} d \tau C_{0}(\tau) \int_{t-\tau}^{t} d t^{\prime} F_{0}\left(t^{\prime}\right)
$$


can be considered as an additional contribution to the fluctuating force. The above expressions for $\gamma^{\star}$ and $F^{\star}(t)$ are valid for time scale $t \gg \tau_{c}$. One can show that in this case $F^{\star}(t)$ is a stationary process. Note that the contribution $F^{\star}(t)$ was overlooked in [20].

The result (44) may be also obtained in a more direct way expanding $p(t-\tau)$ about $t$ :

$$
\begin{aligned}
\lambda^{2} \int_{0}^{t} d \tau M_{1}(\tau) p(t-\tau)= & \lambda^{2} p(t) \int_{0}^{t} d \tau M_{1}(\tau) \\
& -\lambda^{2} \dot{p}(t) \int_{0}^{t} d \tau M_{1}(\tau) \tau+\frac{1}{2 !} \lambda^{2} \ddot{p}(t) \int_{0}^{t} d \tau M_{1}(\tau) \tau^{2}+\cdots
\end{aligned}
$$

To evaluate this expression to order $\lambda^{4}$ one needs derivatives $p^{(n)}(t)$ to order $\lambda^{2}$. The first derivative is given by the linear Langevin equation (40), while for derivatives of higher order Eq.(40) gives: $p^{(n)}(t)=\lambda F_{0}^{(n-1)}(t)+O\left(\lambda^{3}\right)$. Then the above expansion can be transformed into the form (42) with the fluctuating term

$$
\lambda^{3} F^{\star}(t)=\lambda^{3} \int_{0}^{t} d \tau M_{1}(\tau)\left\{F_{0}(t) \tau-\frac{1}{2 !} \dot{F}_{0}(t) \tau^{2}+\cdots\right\} .
$$

Recalling that $M_{1}(t)=\frac{\beta}{m} C_{0}(t)+O\left(\lambda^{2}\right)$ and noticing that

$$
F_{0}(t) \tau-\frac{1}{2 !} \dot{F}_{0}(t) \tau^{2}+\cdots=\int_{0}^{\tau} d t^{\prime} F_{0}\left(t-t^{\prime}\right)=\int_{t-\tau}^{t} d t^{\prime} F_{0}\left(t^{\prime}\right)
$$

one recovers $F^{\star}(t)$ in the form (46).

The above results allow us to write the Langevin equation to order $\lambda^{4}$ in a local form as follows

$$
\frac{d p(t)}{d t}=-\lambda^{2} \gamma_{1} p(t)-\lambda^{4} \gamma_{2} p^{3}(t)-\lambda \xi(t)
$$

Here the fluctuating force is

$$
\xi(t)=F_{0}(t)+\lambda F_{1}(t)+\lambda^{2} F_{2}(t)+\lambda^{2} F^{\star}(t)
$$

and the dissipation coefficients are

$$
\begin{aligned}
\gamma_{1} & =\gamma_{0}+\lambda^{2} \delta \gamma+\lambda^{2} \gamma^{\star} \\
\gamma_{0} & =\frac{\beta}{m} \int_{0}^{\infty} d t C_{0}(t) \\
\delta \gamma & =-\frac{2}{m^{2}} \int_{0}^{\infty} d t C_{1}(t)+\frac{\beta}{m^{2}} \int_{0}^{\infty} d t C_{2}(t) \\
\gamma^{\star} & =\left(\frac{\beta}{m}\right)^{2} \int_{0}^{\infty} d t C_{0}(t) \int_{0}^{\infty} d t C_{0}(t) t \\
\gamma_{2} & =\frac{\beta}{m^{3}} \int_{0}^{\infty} d t C_{1}(t)
\end{aligned}
$$

Recall that the correlation functions $C_{i}(t)$ are defined by Eqs.(28).

\section{FROM LANGEVIN TO FOKKER-PLANCK}

The aim of this section is to construct a Fokker-Planck equation for the distribution function $f(p, t)$ corresponding to the Langevin equation (50). We shall mostly follow the standard procedure [1], but without conventional assumption that the fluctuating force is delta-correlated.

The first step is to assume that $f(p, t)$ obeys a Markovian master equation

$$
\frac{\partial f(p, t)}{\partial t}=\int d p^{\prime}\left\{f\left(p^{\prime}, t\right) w\left(p^{\prime} \rightarrow p\right)-f(p, t) w\left(p \rightarrow p^{\prime}\right)\right\}
$$


Expressing the transition rates $w\left(p_{1} \rightarrow p_{2}\right)$ as a function of the initial state $p_{1}$ and the transition length $\Delta p=p_{2}-p_{1}$, $w\left(p_{1} \rightarrow p_{2}\right)=w\left(p_{1} \mid \Delta p\right)$, the master equation can be written in the form

$$
\begin{aligned}
\frac{\partial f(p, t)}{\partial t} & =\int d(\Delta p)\{f(p-\Delta p, t) w(p-\Delta p \mid \Delta p)-f(p, t) w(p \mid \Delta p)\} \\
& =\int d(\Delta p)\{\Psi(p-\Delta p, \Delta p)-\Psi(p, \Delta p)\},
\end{aligned}
$$

where $\Psi(p, \Delta p)=f(p, t) w(p \mid \Delta p)$. Next, the expansion

$$
\Psi(p-\Delta p, \Delta p)=\Psi(p, \Delta p)+\sum_{n=1}^{\infty} \frac{1}{n !}\left(-\Delta p \frac{\partial}{\partial p}\right)^{n} \Psi(p, \Delta p)
$$

transforms the master equation into the Kramers-Moyal form

$$
\frac{\partial f(p, t)}{\partial t}=\sum_{n=1}^{\infty} \frac{1}{n !}\left(-\frac{\partial}{\partial p}\right)^{n}\left\{\alpha_{n}(p) f(p, t)\right\}
$$

with coefficients $\alpha_{n}$ given by

$$
\alpha_{n}(p)=\int d(\Delta p)(\Delta p)^{n} w(p \mid \Delta p) .
$$

Since transition rates $w$ are usually unknown, it is more convenient to work with another representation for $\alpha_{n}$. Namely, expressing $w$ in terms of the transition probability $T\left(p, t \mid p^{\prime}, t+\tau\right)$

$$
w\left(p \rightarrow p^{\prime}\right)=\lim _{\tau \rightarrow 0} \frac{1}{\tau} T\left(p, t \mid p^{\prime}, t+\tau\right)
$$

one can write the expression (57) for $\alpha_{n}$ in the form

$$
\alpha_{n}(V)=\lim _{\tau \rightarrow 0} \frac{1}{\tau} \int d p^{\prime}\left(p^{\prime}-p\right)^{n} T\left(p, t \mid p^{\prime}, t+\tau\right),
$$

or

$$
\alpha_{n}(p)=\lim _{\tau \rightarrow 0} \frac{1}{\tau}\left\langle[p(t+\tau)-p(t)]^{n}\right\rangle .
$$

This expression may be evaluated integrating the Langevin equation for $p(t)$, but this step needs care. Recall that the Langevin equation (50) corresponds to a coarse-grained description with a time resolution much longer than correlation time of the random force $\tau_{c}$ but much shorter than the characteristic time for the relaxation of the particle's momentum $\tau_{p}$. Then in the above expressions the limit $\tau \rightarrow 0$ should be understood as $\tau_{c} \ll \tau \ll \tau_{p}$. The moments $\left\langle[p(t+\tau)-p(\tau)]^{n}\right\rangle$ in Eq. (59) must be first evaluated in the limit $\tau \gg \tau_{c}$, and only after that the formal operation $\lim _{\tau \rightarrow 0} \frac{1}{\tau}(\cdots)$ must be applied:

$$
\alpha_{n}(p)=\lim _{\tau \rightarrow 0} \frac{1}{\tau}\left\{\lim _{\tau \gg \tau_{c}}\left\langle[p(t+\tau)-p(t)]^{n}\right\rangle\right\} .
$$

In what follows, the coarse-grained limit $\tau \gg \tau_{c}$ for brevity will not be indicated.

Integrating the Langevin equation (50) for $\tau_{c} \ll \tau \ll \tau_{p}$

$$
p(t+\tau)-p(t) \approx-\left[\lambda^{2} \gamma_{1} p(t)+\lambda^{4} \gamma_{2} p^{3}(t)\right] \tau+\lambda \int_{t}^{t+\tau} d t^{\prime} \xi\left(t^{\prime}\right)
$$

and recalling that $\xi(t)$ is a stationary process for $t \gg \tau_{c}$, one obtains from (60):

$$
\begin{aligned}
& \alpha_{1}=-\lambda^{2} \gamma_{1} p-\lambda^{4} \gamma_{2} p^{3} \\
& \alpha_{2}=\lambda^{2} \lim _{\tau \rightarrow 0} \frac{1}{\tau} \int_{0}^{\tau} d t_{1} \int_{0}^{\tau} d t_{2}\left\langle\xi\left(t_{1}\right) \xi\left(t_{2}\right)\right\rangle, \\
& \alpha_{3}=\lambda^{3} \lim _{\tau \rightarrow 0} \frac{1}{\tau} \int_{0}^{\tau} d t_{1} \int_{0}^{\tau} d t_{2} \int_{0}^{\tau} d t_{3}\left\langle\xi\left(t_{1}\right) \xi\left(t_{2}\right) \xi\left(t_{3}\right)\right\rangle, \\
& \alpha_{4}=\lambda^{4} \lim _{\tau \rightarrow 0} \frac{1}{\tau} \int_{0}^{\tau} d t_{1} \int_{0}^{\tau} d t_{2} \int_{0}^{\tau} d t_{3} \int_{0}^{\tau} d t_{4}\left\langle\xi\left(t_{1}\right) \xi\left(t_{2}\right) \xi\left(t_{3}\right) \xi\left(t_{4}\right)\right\rangle .
\end{aligned}
$$


In these expressions the integrals must be taken in the coarse-grained limit $\tau \gg \tau_{c}$.

The next step is to substitute in these expressions the fluctuating force $\xi=F_{0}+\lambda F_{1}+\lambda^{2} F_{2}+\lambda^{2} F^{\star}$ retaining enough terms to get $\alpha_{n}$ to order $\lambda^{4}$. According to (62), the expression for $\alpha_{2}$ requires the correlation $\langle\xi \xi\rangle$ to order $\lambda^{2}$

$$
\begin{aligned}
\left\langle\xi\left(t_{1}\right) \xi\left(t_{2}\right)\right\rangle= & \left\langle F_{0}\left(t_{1}\right) F_{0}\left(t_{2}\right)\right\rangle+\lambda^{2}\left\langle F_{1}\left(t_{1}\right) F_{1}\left(t_{2}\right)\right\rangle+\lambda^{2}\left\langle F_{0}\left(t_{1}\right) F_{0}^{\star}\left(t_{2}\right)\right\rangle+\lambda^{2}\left\langle F_{0}\left(t_{2}\right) F_{0}^{\star}\left(t_{1}\right)\right\rangle \\
& +\lambda^{2}\left\langle F_{0}\left(t_{1}\right) F_{2}\left(t_{2}\right)\right\rangle+\lambda^{2}\left\langle F_{0}\left(t_{2}\right) F_{2}\left(t_{1}\right)\right\rangle,
\end{aligned}
$$

$\alpha_{3}$ requires the correlation $\langle\xi \xi \xi\rangle$ to order $\lambda$,

$$
\left\langle\xi\left(t_{1}\right) \xi\left(t_{2}\right) \xi\left(t_{3}\right)\right\rangle=\lambda\left\langle F_{0}\left(t_{1}\right) F_{0}\left(t_{2}\right) F_{1}\left(t_{3}\right)\right\rangle+\lambda\left\langle F_{0}\left(t_{1}\right) F_{1}\left(t_{2}\right) F_{0}\left(t_{3}\right)\right\rangle+\lambda\left\langle F_{1}\left(t_{1}\right) F_{0}\left(t_{2}\right) F_{0}\left(t_{3}\right)\right\rangle,
$$

and $\alpha_{4}$ requires the correlation $\langle\xi \xi \xi\rangle$ to order $\lambda^{0}$,

$$
\left\langle\xi\left(t_{1}\right) \xi\left(t_{2}\right) \xi\left(t_{3}\right) \xi\left(t_{4}\right)\right\rangle=\left\langle F_{0}\left(t_{1}\right) F_{0}\left(t_{2}\right) F_{0}\left(t_{3}\right) F_{0}\left(t_{4}\right)\right\rangle .
$$

To extract the dependence on $p$ one has to express $F_{1}$ and $F_{2}$ in terms of $p$-independent functions $G_{i}(t)$, see Eq. (23). Then the correlation functions take the forms:

$$
\begin{aligned}
& \left\langle\xi\left(t_{1}\right) \xi\left(t_{2}\right)\right\rangle=C_{0}\left(t_{1}, t_{2}\right)+\lambda^{2} m^{-1}\left\{C_{2}\left(t_{1}, t_{2}\right)+C_{2}\left(t_{2}, t_{1}\right)\right\} \\
& \quad+\lambda^{2}\left(\frac{p}{m}\right)^{2}\left\{C_{1}\left(t_{1}, t_{2}\right)+C_{1}\left(t_{2}, t_{1}\right)+C_{3}\left(t_{2}, t_{1}\right)\right\}+\lambda^{2} \frac{\beta}{m}\left\{C_{6}\left(t_{1}, t_{2}\right)+C_{6}\left(t_{2}, t_{1}\right)\right\}, \\
& \left\langle\xi\left(t_{1}\right) \xi\left(t_{2}\right) \xi\left(t_{3}\right)\right\rangle=\lambda \frac{p}{m}\left\{C_{4}\left(t_{1}, t_{2}, t_{3}\right)+C_{4}\left(t_{1}, t_{3}, t_{2}\right)+C_{4}\left(t_{3}, t_{2}, t_{1}\right)\right\}, \\
& \left\langle\xi\left(t_{1}\right) \xi\left(t_{2}\right) \xi\left(t_{3}\right) \xi\left(t_{4}\right)\right\rangle=C_{0}\left(t_{1}, t_{2}\right) C_{0}\left(t_{3}, t_{4}\right)+C_{0}\left(t_{1}, t_{3}\right) C_{0}\left(t_{2}, t_{4}\right)+C_{0}\left(t_{1}, t_{4}\right) C_{0}\left(t_{2}, t_{3}\right)+C_{5}\left(t_{1}, t_{2}, t_{3}, t_{4}\right) .
\end{aligned}
$$

Here the functions $C_{i}$ are defined as follows

$$
\begin{aligned}
& C_{0}\left(t_{1}, t_{2}\right)=\left\langle\left\langle G_{0}\left(t_{1}\right) G_{0}\left(t_{2}\right)\right\rangle\right\rangle, \\
& C_{1}\left(t_{1}, t_{2}\right)=\int_{0}^{t_{2}} d t^{\prime} \int_{0}^{t^{\prime}} d t^{\prime \prime}\left\langle\left\langle G_{0}\left(t_{1}\right) G_{2}\left(t_{2}, t^{\prime}, t^{\prime \prime}\right)\right\rangle\right\rangle, \\
& C_{2}\left(t_{1}, t_{2}\right)=\int_{0}^{t_{2}} d t^{\prime} \int_{0}^{t^{\prime}} d t^{\prime \prime}\left\langle\left\langle G_{0}\left(t_{1}\right) G_{0}\left(t_{2}-t^{\prime}\right) G_{1}\left(t_{2}, t^{\prime \prime}\right)\right\rangle\right\rangle, \\
& C_{3}\left(t_{1}, t_{2}\right)=\int_{0}^{t_{1}} d t^{\prime} \int_{0}^{t_{2}} d t^{\prime \prime}\left\langle\left\langle G_{1}\left(t_{1}, t^{\prime}\right) G_{1}\left(t_{2}, t^{\prime \prime}\right)\right\rangle\right\rangle, \\
& C_{4}\left(t_{1}, t_{2}, t_{3}\right)=\int_{0}^{t_{3}} d t^{\prime}\left\langle\left\langle G_{0}\left(t_{1}\right) G_{0}\left(t_{2}\right) G_{1}\left(t_{3}, t^{\prime}\right)\right\rangle\right\rangle, \\
& C_{5}\left(t_{1}, t_{2}, t_{3}, t_{4}\right)=\left\langle\left\langle G_{0}\left(t_{1}\right) G_{0}\left(t_{2}\right) G_{0}\left(t_{3}\right) G_{0}\left(t_{4}\right)\right\rangle\right\rangle, \\
& C_{6}\left(t_{1}, t_{2}\right)=\int_{0}^{t_{2}} d t^{\prime} C_{0}\left(t^{\prime}\right) \int_{t_{2}-t^{\prime}}^{t_{2}} d t^{\prime \prime} C_{0}\left(t_{1}, t^{\prime \prime}\right) .
\end{aligned}
$$

Note that the two variable function $C_{0}\left(t_{1}, t_{2}\right)=\left\langle\left\langle G_{0}\left(t_{1}\right) G_{0}\left(t_{2}\right)\right\rangle\right\rangle$ defined above, and the single variable function $C_{0}(t)=\left\langle\left\langle G_{0}(0) G_{0}(t)\right\rangle\right\rangle$ which we used in the previous sections, are related as $C_{0}\left(t_{1}, t_{2}\right)=C_{0}\left(\left|t_{1}-t_{2}\right|\right)$.

Substitution of correlations (66) into (62) leads to the following results

$$
\begin{aligned}
\alpha_{1} & =-\lambda^{2} \gamma_{1} p-\lambda^{4} \gamma_{2} p^{3} \\
\alpha_{2} & =\lambda^{2} \gamma_{3}+\lambda^{4}\left(\frac{p}{m}\right)^{2} \gamma_{4}+\lambda^{4} \frac{1}{m} \gamma_{5}+\lambda^{4} \frac{\beta}{m} \gamma_{6} \\
\alpha_{3} & =\lambda^{4} \frac{p}{m} \gamma_{7} \\
\alpha_{4} & =\lambda^{4} \gamma_{8}
\end{aligned}
$$


Here the dissipation coefficients $\gamma_{1}$ and $\gamma_{2}$, are defined above by equations (52),

$$
\begin{aligned}
\gamma_{3} & =\lim _{\tau \rightarrow 0} \frac{1}{\tau} \int_{0}^{\tau} d t_{1} \int_{0}^{\tau} d t_{2} C_{0}\left(t_{1}, t_{2}\right), \\
\gamma_{4} & =\lim _{\tau \rightarrow 0} \frac{1}{\tau} \int_{0}^{\tau} d t_{1} \int_{0}^{\tau} d t_{2}\left\{2 C_{1}\left(t_{1}, t_{2}\right)+C_{3}\left(t_{1}, t_{2}\right)\right\} \\
\gamma_{5} & =\lim _{\tau \rightarrow 0} \frac{1}{\tau} \int_{0}^{\tau} d t_{1} \int_{0}^{\tau} d t_{2} 2 C_{2}\left(t_{1}, t_{2}\right), \\
\gamma_{6} & =\lim _{\tau \rightarrow 0} \frac{1}{\tau} \int_{0}^{\tau} d t_{1} \int_{0}^{\tau} d t_{2} 2 C_{6}\left(t_{1}, t_{2}\right), \\
\gamma_{7} & =\lim _{\tau \rightarrow 0} \frac{1}{\tau} \int_{0}^{\tau} d t_{1} \int_{0}^{\tau} d t_{2} \int_{0}^{\tau} d t_{3} 3 C_{4}\left(t_{1}, t_{2}, t_{3}\right), \\
\gamma_{8} & =\lim _{\tau \rightarrow 0} \frac{1}{\tau} \int_{0}^{\tau} d t_{1} \int_{0}^{\tau} d t_{2} \int_{0}^{\tau} d t_{3} \int_{0}^{\tau} d t_{4} C_{5}\left(t_{1}, t_{2}, t_{3}, t_{4}\right) .
\end{aligned}
$$

Recall again that in these formulas the integrals must be evaluated in the coarse-grained limit $\tau \gg \tau_{c}$.

Note that in the formula for $\gamma_{8}$ we have discarded the terms involving the products of cumulants, like $\left\langle\left\langle F_{0}\left(t_{1}\right) F_{0}\left(t_{2}\right)\right\rangle\right\rangle\left\langle\left\langle F_{0}\left(t_{3}\right) F_{0}\left(t_{4}\right)\right\rangle\right\rangle$. Such products depend on two time differences, and the corresponding contributions to the four-dimensional time integral in the expression for $\alpha_{4}$ are quadratic in $\tau$. They therefore vanish when the operation $\lim _{\tau \rightarrow 0} \frac{1}{\tau}(\cdots)$ is applied.

\section{GENERALIZED FOKKER-PLANCK EQUATION}

Substituting the results (68) for $\alpha_{n}$ into the Kramers-Moyal expansion (56) one arrives at the Fokker-Planck equation of order $\lambda^{4}$ in the following form

$$
\frac{\partial f(p, t)}{\partial t}=\left\{\lambda^{2} D_{2}+\lambda^{4} D_{4}+\lambda^{4} D_{2}^{\star}\right\} f(p, t)
$$

Here the differential operators $D_{2}$ and $D_{4}$ have the same structure as for the van Kampen equation (6),

$$
\begin{aligned}
& D_{2}=a_{1} \frac{\partial}{\partial p} p+a_{2} \frac{\partial^{2}}{\partial p^{2}} \\
& D_{4}=b_{1} \frac{\partial}{\partial p} p+b_{2} \frac{\partial}{\partial p} p^{3}+b_{3} \frac{\partial^{2}}{\partial p^{2}}+b_{4} \frac{\partial^{2}}{\partial p^{2}} p^{2}+b_{5} \frac{\partial^{3}}{\partial p^{3}} p+b_{6} \frac{\partial^{4}}{\partial p^{4}}
\end{aligned}
$$

The difference with the van Kampen equation is the presence of the operator $D_{2}^{\star}$, originating from non-Markovian corrections $\gamma^{\star}$ and $F^{\star}$. It has the same structure as $D_{2}$

$$
D_{2}^{\star}=c_{1} \frac{\partial}{\partial p} p+c_{2} \frac{\partial^{2}}{\partial p^{2}}
$$

but as we shall see, scales differently with the bath density $n$.

For the operator $D_{2}$ the coefficients are $a_{1}=\gamma_{0}$ and $a_{2}=\frac{1}{2} \gamma_{3}$,

$$
\begin{aligned}
& a_{1}=\frac{\beta}{m} \int_{0}^{\infty} d t C_{0}(t), \\
& a_{2}=\frac{1}{2 \tau} \int_{0}^{\tau} d t_{1} \int_{0}^{\tau} d t_{2} C_{0}\left(t_{1}, t_{2}\right) .
\end{aligned}
$$

The expression for $a_{2}$ may be simplified recalling that $C_{0}\left(t_{1}, t_{2}\right)=C_{0}\left(\left|t_{1}-t_{2}\right|\right)$ and using the coarse-grained limit $\tau \gg \tau_{c}$ :

$$
a_{2}=\frac{1}{\tau} \int_{0}^{\tau} d t_{2} \int_{0}^{t_{2}} d t_{1} C_{0}\left(t_{1}, t_{2}\right)=\frac{1}{\tau} \int_{0}^{\tau} d t_{2}\left(\tau-t_{2}\right) C_{0}\left(t_{2}\right) \rightarrow \int_{0}^{\infty} d t C_{0}(t)
$$


This gives the conventional relation

$$
\frac{a_{1}}{a_{2}}=\frac{\beta}{m}
$$

which guarantees that the Maxwellian distribution $f_{M}(p)=C \exp \left(-\beta p^{2} / 2 m\right)$ is stationary for the operator $D_{2}$ : $D_{2} f_{M}(p)=0$.

For the operator $D_{4}$ the coefficients are $b_{1}=\delta \gamma, b_{2}=\gamma_{2}, b_{3}=\gamma_{4} / 2 m^{2}, b_{4}=\gamma_{5} / 2 m, b_{5}=\gamma_{7} / 3 ! m$, and $b_{6}=\gamma_{8} / 4$ ! Using the results (52) and (69) for $\gamma_{i}$, one can write the coefficients $b_{i}$ in terms of correlation functions as follows:

$$
\begin{aligned}
& b_{1}=\frac{2}{m^{2}} \int_{0}^{\infty} d t C_{1}(t)+\frac{\beta}{m^{2}} \int_{0}^{\infty} d t C_{2}(t), \\
& b_{2}=\frac{\beta}{m^{3}} \int_{0}^{\infty} d t C_{1}(t) \\
& b_{3}=\frac{1}{2 m^{2}} \lim _{\tau \rightarrow 0} \frac{1}{\tau} \int_{0}^{\tau} d t_{1} \int_{0}^{\tau} d t_{2}\left\{2 C_{1}\left(t_{1}, t_{2}\right)+C_{3}\left(t_{1}, t_{2}\right)\right\} \\
& b_{4}=\frac{1}{m} \lim _{\tau \rightarrow 0} \frac{1}{\tau} \int_{0}^{\tau} d t_{1} \int_{0}^{\tau} d t_{2} C_{2}\left(t_{1}, t_{2}\right) \\
& b_{5}=\frac{1}{2 m} \lim _{\tau \rightarrow 0} \frac{1}{\tau} \int_{0}^{\tau} d t_{1} \int_{0}^{\tau} d t_{2} \int_{0}^{\tau} d t_{3} C_{4}\left(t_{1}, t_{2}, t_{3}\right), \\
& b_{6}=\frac{1}{4 !} \lim _{\tau \rightarrow 0} \frac{1}{\tau} \int_{0}^{\tau} d t_{1} \int_{0}^{\tau} d t_{2} \int_{0}^{\tau} d t_{3} \int_{0}^{\tau} d t_{4} C_{5}\left(t_{1}, t_{2}, t_{3}, t_{4}\right) .
\end{aligned}
$$

Here the functions $C_{i}$ are defined by relations (28) and (67), and the integrals must be evaluated in the coarse-grained limit $\tau \gg \tau_{c}$.

We did not attempt to give a general prove that $D_{4}$, with coefficients given above, satisfies the stationary relation $D_{4} f_{M}(p)=0$. Instead, in the next section we evaluate coefficients $b_{i}$ explicitly for the exactly solvable generalized Rayleigh model. In this case $D_{4}$ is found to be the same as for the van Kampen equation (6) , and therefore the relation $D_{4} f_{M}(p)=0$ is satisfied.

Consider at last the coefficients for the operator $D_{2}^{\star}$ :

$$
\begin{aligned}
& c_{1}=\gamma^{\star}=\left(\frac{\beta}{m}\right)^{2} \int_{0}^{\infty} d t C_{0}(t) \int_{0}^{\infty} d t C_{0}(t) t, \\
& c_{2}=\frac{\beta}{2 m} \gamma_{6}=\frac{\beta}{m} \lim _{\tau \rightarrow 0} \frac{1}{\tau} \int_{0}^{\tau} d t_{1} \int_{0}^{\tau} d t_{2} C_{6}\left(t_{1}, t_{2}\right) .
\end{aligned}
$$

It is proved in the Appendix that

$$
\frac{c_{1}}{c_{2}}=\frac{\beta}{2 m} .
$$

Because of the factor two in the denominator the Maxwellian distribution $f_{M} \sim \exp \left(-\beta p^{2} / 2 m\right)$ is not stationary for the operator $D_{2}^{\star}, D_{2}^{\star} f_{M}(p) \neq 0$. The validity of the relation (79) can be also verified directly, for instance, for $C_{0}(t) \sim \exp \left(-t / \tau_{c}\right)$. One might hope that keeping terms of even higher orders would restore Maxwellian equilibrium. However, the distribution $f_{M}(p)$ does not depend on $\lambda$, which suggests that it must satisfy each term of the $\lambda$-expansion separately.

Note that coefficients $c_{1}$ and $c_{2}$ are quadratic in cumulants $C_{0}(t)=\left\langle\left\langle F_{0}\left(t_{1}\right) F_{0}\left(t_{2}\right)\right\rangle\right\rangle$, and therefore quadratic in the bath density, $D_{2}^{\star} \sim n^{2}$. In contrast, the operators $D_{2}$ and $D_{4}$ are linear in $n$. Therefore, in the low-density limit $D_{2}^{\star}$ may be neglected, and the generalized FPE (70) is reduced to the van Kampen equation (6). On the other hand, one may expect that for sufficiently high density of the bath the operator $D_{4} \sim n$ may be neglected compared to $D_{2}^{\star} \sim n^{2}$, which results in a conventional FPE

$$
\frac{\partial f(p, t)}{\partial t}=\lambda^{2}\left\{A_{1} \frac{\partial}{\partial p} p+A_{2} \frac{\partial^{2}}{\partial p^{2}}\right\} f(p, t),
$$

but with modified coefficients

$$
A_{1}=a_{1}+\lambda^{2} c_{1}, A_{2}=a_{2}+\lambda^{2} c_{2}
$$


The stationary solution of this equation is the Maxwellian distribution $\exp \left[-\beta^{\star} p^{2} / 2 m\right]$ with the inverse temperature

$$
\beta^{\star}=\frac{m A_{1}}{A_{2}}=m \frac{a_{1}+\lambda^{2} c_{1}}{a_{2}+\lambda^{2} c_{2}},
$$

which is smaller than that for the bath, $\beta$. Indeed, recalling that $a_{1} / a_{2}=\beta / m$ and $c_{1} / c_{2}=\beta / 2 m$, one obtains to order $\lambda^{2}$

$$
\frac{\beta^{\star}}{\beta}=1-\lambda^{2} \frac{c_{1}}{a_{1}}=1-\lambda^{2} \frac{\beta}{m} \int_{0}^{\infty} d t C_{0}(t) t .
$$

Needless to say, the prediction that the temperature of a Brownian particle is higher than the temperature of the bath is in contradiction with basic assumptions of equilibrium statistical physics and must be subjected to a thorough scrutiny. It might be instructive to consider a specific model.

\section{GENERALIZED RAYLEIGH MODEL}

For the generalized Rayleigh model [20] it is possible to evaluate the coefficients in the nonlinear Langevin equation (50) and generalized Fokker-Planck equation (70) analytically. In this model the bath molecules do not interact with each other, while the Brownian particle interact with molecules not through instantaneous collisions, as in the original Rayleigh model [3, 4, 5], but via a continuous parabolic repulsive potential. Namely, when the distance between a molecule and the particle $\left|x_{i}-X\right|$ is larger than a given length $R$ the molecule moves freely. But when the molecule enters the "interaction zone" $\left|x_{i}-X\right|<R$, it experiences a repulsive parabolic potential $\frac{1}{2} k\left(x_{i}-\tilde{X}\right)^{2}$, where $\tilde{X}=X-R$ for a molecule approaching the particle from the left, and $\tilde{X}=X+R$ for a molecule from the right.

As was shown in [20], for this model all relevant correlation functions can be evaluated exactly. In particular, the correlation functions $C_{i}(t)$, Eqs. (28), which determine dissipative coefficients in the nonlinear Langevin equation (50), read

$$
\begin{aligned}
& C_{0}(t)=\omega \nu p_{T}^{3} m^{-1} \xi_{0}(\omega t), \\
& C_{1}(t)=\omega \nu p_{T} m \xi_{1}(\omega t), \\
& C_{2}(t)=-\omega \nu p_{T}^{3} \xi_{2}(\omega t) .
\end{aligned}
$$

Here $\omega=\sqrt{k / m}$ is the inverse collision time, $\nu$ is the number of molecules per unit length, $p_{T}=\sqrt{m / \beta}$ is the thermal momentum of a molecule, and dimensionless functions $\xi_{i}(x)$ are

$$
\begin{aligned}
& \xi_{0}(x)=\frac{2}{\sqrt{2 \pi}} \theta(\pi-x)\{\sin x+(\pi-x) \cos x\}, \\
& \xi_{1}(x)=\frac{1}{\sqrt{2 \pi}} \theta(\pi-x) \sin ^{3} x, \\
& \xi_{2}(x)=\frac{1}{\sqrt{2 \pi}} \theta(\pi-x)\left\{\sin ^{3} x+x(\pi-x) \sin x\right\},
\end{aligned}
$$

where $\theta(x)$ is the Heaviside unit step function. Substitution of these results into Eqs. (52) gives the dissipative constants for the nonlinear Langevin equation (50)

$$
\frac{d p(t)}{d t}=\lambda^{2} \gamma_{1} p(t)-\lambda^{4} \gamma_{2} p^{3}(t)-\lambda \xi(t)
$$

in the form

$$
\begin{aligned}
\gamma_{1} & =\gamma_{0}+\lambda^{2} \delta \gamma+\lambda^{2} \gamma^{\star}, \\
\gamma_{0} & =\frac{\nu p_{T}}{m} \int_{0}^{\infty} d x \xi_{0}(x)=\frac{8}{\sqrt{2 \pi}} \frac{\nu p_{T}}{m}, \\
\delta \gamma & =-\frac{\nu p_{T}}{m} \int_{0}^{\infty} d x\left[2 \xi_{1}(x)+\xi_{2}(x)\right]=-\frac{8}{\sqrt{2 \pi}} \frac{\nu p_{T}}{m}, \\
\gamma^{\star} & =\frac{\nu^{2} p_{T}^{2}}{\omega m^{2}} \int_{0}^{\infty} d x \xi_{0}(x) \int_{0}^{\infty} d x \xi_{0}(x) x=8 \frac{\nu^{2} p_{T}^{2}}{\omega m^{2}}, \\
\gamma_{2} & =\frac{\nu}{m p_{T}} \int_{0}^{\infty} d x \xi_{1}(x)=\frac{4}{3 \sqrt{2 \pi}} \frac{\nu}{m p_{T}} .
\end{aligned}
$$


Consider now the generalized FPE (170)

$$
\frac{\partial f(p, t)}{\partial t}=\left\{\lambda^{2} D_{2}+\lambda^{4} D_{4}+\lambda^{4} D_{2}^{\star}\right\} f(p, t)
$$

The coefficients in the operators $D_{i}$ are determined by correlations (67). They can be evaluated in the same manner as the correlations (84). Then one can show that the operators $D_{2}$ and $D_{4}$ coincide with those for the original Rayleigh model with instantaneous binary collisions and are given by Eqs. (5) and (7), respectively.

The operator $D_{2}^{\star}$ originates from non-Markovian corrections and does not appear in the generalized FPE for the original Rayleigh model with instantaneous collisions. It has the form

$$
D_{2}^{\star}=c_{1} \frac{\partial}{\partial p} p+c_{2} \frac{\partial^{2}}{\partial p^{2}}
$$

with coefficients

$$
c_{1}=\gamma^{\star}=8 \frac{\nu^{2} p_{T}^{2}}{\omega m^{2}}, \quad c_{2}=16 \frac{\nu^{2} p_{T}^{4}}{\omega m^{2}} .
$$

In accord with the general prediction (79), one observes that $c_{1} / c_{2}=\beta / 2 m$, and therefore the Maxwellian distribution $f_{M}(p)=C \exp \left(-\beta p^{2} / 2 m\right)$ is not stationary for the operator, $D_{2}^{\star} f_{M}(p) \neq 0$.

Recall that $D_{4}$ and $D_{2}^{\star}$ scale differently with the molecular density $\nu: D_{4} \sim \nu, D_{2}^{\star} \sim \nu^{2}$. This suggests that for sufficiently high density, the operator $D_{4}$ can be dropped. Inspecting Eqs.(7) and (90), one finds that the ratio of terms generated by $D_{2}^{\star}$ to those produced by $D_{4}$ is of order

$$
N=\frac{\nu p_{T}}{\omega m}
$$

This is an important parameter of the problem and has a meaning of average number of molecules simultaneously interacting with the particle. For the case $N \ll 1$, corresponding to the limit of binary collision, the operator $D_{2}^{\star}$ can be neglected, and the generalized FPE is reduced to the van Kampen equation (6). The opposite limit $N \gg 1$ corresponds to multiple collisions. In this case the operator $D_{2}^{\star}$ is expected to be more important than $D_{4}$, and the generalized FPE to be reduced to the form (80). As discussed in the previous section, this equation has a Maxwellian solution with the inverse temperature $\beta^{\star}$ smaller than that for the bath $\beta$. The ratio $\beta^{\star} / \beta$ is given by Eq. (83), which for the given model takes the form

$$
\frac{\beta^{\star}}{\beta}=1-\sqrt{2 \pi} N \lambda^{2}
$$

We attempted to verify this prediction in a numerical experiment using a simulation setup similar to that described in detail in [9]. In our simulation two Poissonian sources of in-going molecules are located sufficiently far from the particle to mimic an infinite thermal bath with a Maxwellian velocity distribution of molecules. The particle's position is initially fixed and the bath is allowed to equilibrate in the field of the fixed particle for a time $t_{e q}$. Then, at the moment $t=0$ the particle is released with an initial velocity $V(0)$, and the relaxation of the mean-square velocity $\left\langle V^{2}(t)\right\rangle$ towards its stationary value is calculated. Fig. 1 shows the result for the mass ratio $\lambda^{2}=10^{-3}$ and the density of the bath corresponding to $N=100$. The velocity is in units of the thermal velocity of bath molecules $v_{T}=1 / \sqrt{m \beta}$, so that according to equilibrium statistical mechanics $\left\langle V^{2}(t)\right\rangle$ should approach the equilibrium value $\lambda^{2}=0.001$. Instead, the stationary mean-square velocity of the particle appears to be slightly higher. However, the exceeding is less than 0.1 percent which is orders of magnitude lower than predicted by Eq.(92). The smallness of the alleged deviation from the Maxwellian average requires very large number of sampling runs, which in our case was about $3 \cdot 10^{7}$. Also, the result seems to be sensitive to the value of the integration time step $\Delta t$. We used the velocity Verlet algorithm with $\Delta t \sim 0.001$ in units of the collision time $\omega^{-1}$. Simulations with larger $\Delta t$ show no signs of deviations from Maxwellian statistics.

\section{CONCLUDING REMARKS}

In this paper the Fokker-Planck equation for the Brownian particle is attempted to be derived beyond the lowest order in the mass ratio $\lambda^{2}=m / M$. Unlike the approach of van Kampen and Oppenheim [15], the projection operator technique was applied to the equation of motion for the particle, rather than to the Liouville equation. The results seem both encouraging and controversial. 


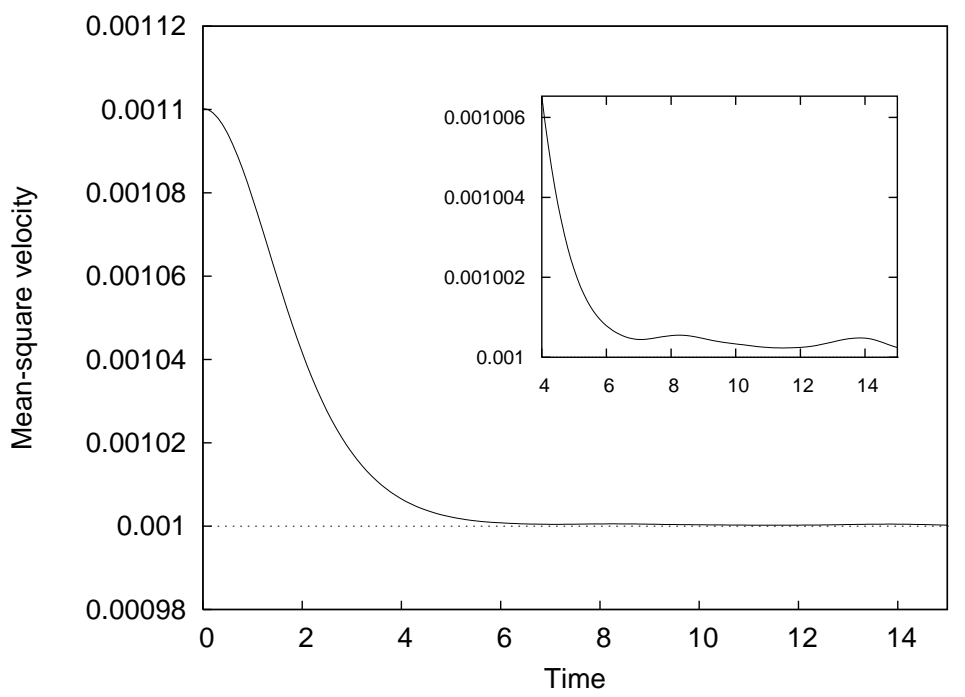

FIG. 1: The relaxation of the mean-square velocity of a Brownian particle $\left\langle V^{2}(t)\right\rangle$ for the generalized Rayleigh model. Parameters of simulations are $\lambda^{2}=0.001, N=100, t_{e q}=7.5$. Time is in units of the collision time $\omega^{-1}$, and velocity is in units of the thermal velocity of bath molecules $v_{T}$. Zoomed data shown in the inset suggest that the stationary value exceeds the Maxwellian average $\left\langle V^{2}\right\rangle=\lambda^{2}=0.001$.

On the one hand, the van Kampen equation, originally designed for the special case of instantaneous collisions, is recovered from first principles for a low-density bath. Therefore, in the Markovian limit the method is correct.

On the other hand, it is found that in general case the equation contains the additional operator $\lambda^{4} D_{2}^{\star}$ originating from non-Markovian corrections which are inevitable for any model with finite collision time. These corrections are found to make the stationary solution non-Maxwellian, $D_{2}^{\star} f_{M}(p) \neq 0$, which is, of course, a very disturbing result. Brownian motion of a massive particle coupled to an infinite bath in thermal equilibrium, with the Maxwellian velocity distribution for bath molecules, is often considered as a classical example of an ergodic process: all accessible microstates are equally probable over a long period of time, and therefore the equilibrium state is the Gibbs canonical ensemble. To order $\lambda^{2}$ this anticipation is supported by solutions of both Langevin and Fokker-Planck equations. To order $\lambda^{4}$, the original Rayleigh model with instantaneous collisions leads to the van Kampen equation which also has the Maxwellian stationary solution. Note that in this last case the ergodic behavior can not be deduced from the Central Limit Theorem or the assumption of Gaussian noise imposed on the Langevin equation (which predict the FPE of second order). Instead, one can show that the van Kampen equation is equivalent to a master equation with transition rates obeying the detailed balance condition [4]. As well known, in this case the applicability of Boltzmann-Gibbs statistics can be readily proved [2]. However, whether or not the detailed balance holds in general case is not known, and a truly dynamical justification of Boltzmann-Gibbs statistics still seems to be missing [23]. A number of generalizations of Boltzmann-Gibbs statistics has been discussed in recent years, including those derived directly from underlying dynamics (see for instance [24, 25, 26] and references therein). However, these generalizations usually imply an essential departure from basic assumptions of Boltzmann-Gibbs statistical mechanics such as inelastic collisions, nonextensivity, Lévy statistics for the bath, etc. In contrast, the result $D_{2}^{\star} f_{M}(p) \neq 0$ originates merely from finite duration of collisions, which suggests deviations from Boltzmann-Gibbs statistics under a much wider range of conditions.

This radical prediction is in contradiction with the results of van Kampen and Oppenheim [15]. They obtained a generalized FPE in which the operator $D_{2}^{*}$ (in our notations) has the form of $D_{2}$ squared, and therefore the stationary solution is Maxwellian. This casts some scepticism about the ability of the perturbation approach exploited in this paper to treat non-Markovian effects. The method is based on the seemingly straightforward assumption of wide separation of time scales for the particle's momentum $\tau_{p} \sim \lambda^{-2}$ and for correlation functions of the fluctuating force $\tau_{c} \sim \lambda^{0}$. However, for a finite $\lambda$, the relation $\tau_{p} \gg \tau_{c}$ is not necessarily true, even if the coupling with slow bath's collective modes is negligible [14]. On the other hand, the van Kampen-Oppenheim approach also relies on the assumption $\tau_{p} \gg \tau_{c}$, and it is not clear why the two approaches give different results. Numerical simulations of the generalized Rayleigh model apparently suggest some deviation from the Maxwellian statistics, but much smaller than the theory predicts. It should be stressed, however, that the presented theory is asymptotic and implies the weak coupling limit, which is difficult to approach in a numerical experiment. One may hope that further, more accurate numerical modeling would shed some light on these questions. 


\section{ACKNOWLEDGMENT}

This work was partly supported by NSERC.

\section{Appendix}

In this Appendix we prove the relation (79): $c_{1} / c_{2}=\beta / 2 m$. Using the definition of $c_{1}$ and $c_{2}$, Eq. (78), the relation can be written in the form

$$
\int_{0}^{\tau} d t_{1} \int_{0}^{\tau} d t_{2} C_{6}\left(t_{1}, t_{2}\right) \underset{\tau \gg \tau_{c}}{\longrightarrow} 2 \tau \int_{0}^{\infty} d t C_{0}(t) \int_{0}^{\infty} d t t C_{0}(t)
$$

or taking the derivative

$$
\int_{0}^{\tau} d t C_{6}(t, \tau)+\int_{0}^{\tau} d t C_{6}(\tau, t) \underset{\tau \gg \tau_{c}}{\longrightarrow} 2 \int_{0}^{\infty} d t C_{0}(t) \int_{0}^{\infty} d t t C_{0}(t) .
$$

Asymptotic relations (A1) and (A2) are equivalent but the latter is easier to prove. Let us evaluate two integrals in the left hand side of the relation (A2).

Consider the first integral $I_{1}=\int_{0}^{\tau} d t C_{6}(t, \tau)$. Recalling the definition of $C_{6}$

$$
C_{6}\left(t_{1}, t_{2}\right)=\int_{0}^{t_{2}} d t^{\prime} C_{0}\left(t^{\prime}\right) \int_{t_{2}-t^{\prime}}^{t_{2}} d t^{\prime \prime} C_{0}\left(\left|t_{1}-t^{\prime \prime}\right|\right)
$$

one can write

$$
\begin{aligned}
I_{1} & =\int_{0}^{\tau} d t \int_{0}^{\tau} d t^{\prime} C_{0}\left(t^{\prime}\right) \int_{\tau-t^{\prime}}^{\tau} d t^{\prime \prime} C_{0}\left(\left|t-t^{\prime \prime}\right|\right) \\
& =\int_{0}^{\tau} d t^{\prime} C_{0}\left(t^{\prime}\right) \int_{\tau-t^{\prime}}^{\tau} d t^{\prime \prime}\left\{\int_{0}^{t^{\prime \prime}} d t C_{0}\left(t^{\prime \prime}-t\right)+\int_{t^{\prime \prime}}^{\tau} d t C_{0}\left(t-t^{\prime \prime}\right)\right\} \\
& =\int_{0}^{\tau} d t^{\prime} C_{0}\left(t^{\prime}\right) \int_{\tau-t^{\prime}}^{\tau} d t^{\prime \prime} \int_{0}^{t^{\prime \prime}} d t^{\prime \prime \prime} C_{0}\left(t^{\prime \prime \prime}\right)+\int_{0}^{\tau} d t^{\prime} C_{0}\left(t^{\prime}\right) \int_{\tau-t^{\prime}}^{\tau} d t^{\prime \prime} \int_{0}^{\tau-t^{\prime \prime}} d t^{\prime \prime \prime} C_{0}\left(t^{\prime \prime \prime}\right) .
\end{aligned}
$$

Introducing the function

$$
\Phi(t)=\int_{0}^{t} d t^{\prime} C_{0}\left(t^{\prime}\right)
$$

the above expression can be written as follows

$$
I_{1}=\int_{0}^{\tau} d t^{\prime} C_{0}\left(t^{\prime}\right) \int_{\tau-t^{\prime}}^{\tau} d t^{\prime \prime} \Phi\left(t^{\prime \prime}\right)+\int_{0}^{\tau} d t^{\prime} C_{0}\left(t^{\prime}\right) \int_{0}^{t^{\prime}} d t^{\prime \prime} \Phi\left(t^{\prime \prime}\right) .
$$

Noticing that $\Phi^{\prime}(t)=C_{0}(t)$, it is convenient to integrate the last term by parts:

$$
I_{1}=\int_{0}^{\tau} d t^{\prime} C_{0}\left(t^{\prime}\right) \int_{\tau-t^{\prime}}^{\tau} d t^{\prime \prime} \Phi\left(t^{\prime \prime}\right)+\Phi(\tau) \int_{0}^{\tau} d t \Phi(t)-\int_{0}^{\tau} d t \Phi^{2}(t) .
$$

Consider now the second integral in the left hand side of (A2)

$$
I_{2}=\int_{0}^{\tau} d t C_{6}(\tau, t)=\int_{0}^{\tau} d t \int_{0}^{t} d t^{\prime} C_{0}\left(t^{\prime}\right) \int_{t-t^{\prime}}^{t} d t^{\prime \prime} C_{0}\left(\left|\tau-t^{\prime \prime}\right|\right)
$$

Since $t^{\prime \prime}<t<\tau$ the symbol of absolute value may be omitted, and a change of variables gives

$$
I_{2}=\int_{0}^{\tau} d t^{\prime} \int_{t^{\prime}}^{\tau} d t^{\prime \prime} C_{0}\left(t^{\prime \prime}-t^{\prime}\right) \int_{t^{\prime}}^{t^{\prime \prime}} d t^{\prime \prime \prime} C_{0}\left(t^{\prime \prime \prime}\right)=-\int_{0}^{\tau} d t^{\prime} t^{\prime} \frac{d}{d t^{\prime}} \int_{t^{\prime}}^{\tau} d t^{\prime \prime} C_{0}\left(t^{\prime \prime}-t^{\prime}\right) \int_{t^{\prime}}^{t^{\prime \prime}} d t^{\prime \prime \prime} C_{0}\left(t^{\prime \prime \prime}\right)
$$


Evaluation of the derivatives gives

$$
I_{2}=\int_{0}^{\tau} d t^{\prime} t^{\prime} C_{0}\left(\tau-t^{\prime}\right) \int_{t^{\prime}}^{\tau} d t^{\prime \prime} C_{0}\left(t^{\prime \prime}\right)+\int_{0}^{\tau} d t^{\prime} t^{\prime} C_{0}\left(t^{\prime}\right) \Phi\left(\tau-t^{\prime}\right)-\int_{0}^{\tau} d t^{\prime} t^{\prime} \int_{t^{\prime}}^{\tau} d t^{\prime \prime} C_{0}\left(t^{\prime \prime}-t^{\prime}\right) C_{0}\left(t^{\prime \prime}\right) .
$$

Integrating by parts, the last term in this expression can be presented in the form

$$
-\int_{0}^{\tau} d t^{\prime} t^{\prime} \int_{t^{\prime}}^{\tau} d t^{\prime \prime} C_{0}\left(t^{\prime \prime}-t^{\prime}\right) C_{0}\left(t^{\prime \prime}\right)=-\Phi(\tau) \int_{0}^{\tau} d t^{\prime} \Phi\left(t^{\prime}\right)+\int_{0}^{\tau} d t \Phi^{2}(t)
$$

so finally for $I_{2}$ one obtains

$$
I_{2}=\int_{0}^{\tau} d t^{\prime} t^{\prime} C_{0}\left(\tau-t^{\prime}\right) \int_{t^{\prime}}^{\tau} d t^{\prime \prime} C_{0}\left(t^{\prime \prime}\right)+\int_{0}^{\tau} d t t C_{0}(t) \Phi(\tau-t)-\Phi(\tau) \int_{0}^{\tau} d t \Phi(t)+\int_{0}^{\tau} d t \Phi^{2}(t) .
$$

Together with (A3) this gives for the left hand side of relation (A2)

$$
I_{1}+I_{2}=\int_{0}^{\tau} d t^{\prime} t^{\prime} C_{0}\left(\tau-t^{\prime}\right) \int_{t^{\prime}}^{\tau} d t^{\prime \prime} C_{0}\left(t^{\prime \prime}\right)+\int_{0}^{\tau} d t^{\prime} C_{0}\left(t^{\prime}\right) \int_{\tau-t^{\prime}}^{\tau} d t^{\prime \prime} \Phi\left(t^{\prime \prime}\right)+\int_{0}^{\tau} d t t C_{0}(t) \Phi(\tau-t) .
$$

In the limit $\tau \gg \tau_{c}$ the first term in this expression vanishes, while the second and the third terms both equal $\Phi(\infty) \int_{0}^{\infty} d t t C_{0}(t)$ :

$$
I_{1}+I_{2} \underset{\tau \gg \tau_{c}}{\longrightarrow} 2 \Phi(\infty) \int_{0}^{\infty} d t t C_{0}(t)
$$

But this is just the right hand side of the relation (A2), which thus is proved.

[1] H. Risken, The Fokker-Planck Equation, Springer, Berlin, 1989.

[2] N.G. van Kampen, Stochastic processes in physics and chemistry, North-Holland, Amsterdam, 1981.

[3] N. G. van Kampen, Can. J. Phys. 39, 551 (1961).

[4] C.T.J. Alkemade, N.G. van Kampen, and D.K.C. MacDonald, Proc. R. Soc. 271A, 449 (1963).

[5] A.V. Plyukhin, Physica A 351, 198 (2005).

[6] Ch. Gruber and J. Piasecki, Physica A 268, 412 (1999); E. Kestemont, C. Van den Broeck, and M. M. Mansour, Europhys. Lett 49, 143 (2000); T. Munakata and H. Ogawa, Phys. Rev. E 64, 036119 (2001); A.V. Plyukhin and J. Schofield, Phys. Rev. E 69, 021112 (2004).

[7] P. Meurs, C. Van den Broeck, and A. Garcia, Phys. Rev. E 70, 051109 (2004).

[8] C. Van den Broeck, R. Kawai, and P. Meurs, Phys. Rev. Lett. 93, 090601 (2004); C. Van den Broeck, P. Meurs and R. Kawai, New J. Phys. 7, 10 (2005).

[9] A.V. Plyukhin and A.M. Froese, Phys. Rev. E 76, 031121 (2007).

[10] M. F. Gelin and D. S. Kosov, J. Chem. Phys. 126, 244501 (2007).

[11] J. Lebowitz and E. Rubin, Phys. Rev. 131, 2381 (1963); P. Résibois and H. Davis, Physica 30, 1077 (1963); J. Lebowitz and P. Résibois, Phys. Rev. A 139, 1101 (1965).

[12] H. Mori, H. Fujisaka, and H. Shigematsu, Prog. Theor. Phys. 51, 109 (1974).

[13] E.L. Chang, R.M. Mazo, and J.T. Hynes, Mol. Phys. 28, 997 (1974).

[14] J.T. Hynes, R. Kapral, and M. Weinberg, Physica A 80, 105 (1975).

[15] N. van Kampen and I. Oppenheim, Physica A 138, 231 (1986).

[16] J. Seke, J. Phys. A 23, L6 (1990); ibid. 24, 2121 (1991).

[17] A.V. Plyukhin, Europhys. Lett. 75, 15 (2006).

[18] M. Lissia and P. Quarati, Europhys. News 36, 211 (2005).

[19] G. Kaniadakis and P. Quarati, Physica A 192, 677 (1993).

[20] A.V. Plyukhin and J. Schofield, Phys. Rev. E 68, 041107 (2003).

[21] P. Mazur and I. Oppenheim, Physica 50, 241 (1970).

[22] R.M. Mazo, Brownian motion, Oxford University Press, 2002, Ch. 11.

[23] E.G.D. Cohen, Physica A, 305, 19 (2002).

[24] M. Annunziato, P. Grigolini, and B.J. West, Phys. Rev. E 64, 011107 (2001).

[25] E. Barkai, J. Stat. Phys. 115, 1537 (2004).

[26] J.D. Bao, P. Hänggi, and Y.Z. Zhuo, Phys. Rev. E 72, 061107 (2005). 